\title{
ORIGINAL
}

\section{PREVALENCIA Y FACTORES ASOCIADOS DE LAAUTOMONITORIZACIÓN GLUCÉMICA EN PACIENTES DIABÉTICOS TIPO 2 NO TRATADOS CON INSULINA EN LA COMUNIDAD VALENCIANA (*)}

\author{
Diego Cano-Blanquer (1,2), Pedro Cervera-Casino (1,3), Salvador Peiró-Moreno (4), Mónica \\ Mateu-García (1,5), Amparo Barreda-Aznar (1,6) y Grupo de Estudio de la Automonitorización \\ Glucémica $(\dagger)$.
}

(1) Sociedad Valenciana de Farmacéuticos de Atención Primaria.

(2) Departamento de Salud de València-Clínic-La Malva-rosa. Agencia Valenciana de Salud (AVS). Valencia.

(3) Departamento de Salud de La Marina Alta. Agencia Valenciana de Salud. Denia.

(4) Centro Superior de Investigación en Salud Pública (CSISP-FISABIO). Red de Investigación en Servicios de Salud en Enfermedades Crónicas (REDISSEC). Valencia.

(5) Departamento de Salud de Vinaròs. Agencia Valenciana de Salud. Vinaróz.

(6) Departamento de Salud de Castelló. Agencia Valenciana de Salud. Castellón.

$\left.{ }^{*}\right)$ Este proyecto obtuvo una ayuda concurrente en la convocatoria para estudios de investigación en evaluación de tecnologías sanitarias de la Conselleria de Sanitat de la Generalitat Valenciana (Ev Tec: 027/2009).

(†) Anexo 1

Los autores declaran que no existen conflictos de intereses.

\section{RESUMEN}

Fundamentos: La utilidad de la automonitorización de la glucemia (AMGS) en pacientes con diabetes tipo 2 (DM2) no tratados con insulina (NTI) es objeto de controversia.. Los objetivos de este trabajo son describir la prevalencia de AMGS en sujetos con DM2 NTI en la Comunidad Valenciana y analizar los factores asociados a su a su uso.

Método: Estudio transversal en 83 consultas de atención primaria mediante revisión de la historia clínica electrónica y entrevista a 573 sujetos con DM2-NTI. Se analizó la asociación entre la indicación de AMGS y las características del paciente, médico y organizativas.

Resultados: 289 (50,4\%) de los pacientes con DM2-NTI utilizaban AMGS. En el análisis multivariante el uso de AMGS se asoció a la menor edad (OR:2,3 para 65-84 y 6,0 para $<65$, vs $85+$ años), duración de la diabetes (OR:2,2 para 10-15 años vs 0-5 años), sedentarismo (OR:1,6), obesidad (OR:1,5), situación laboral, valor de la hemoglobina glicosilada (OR:1,9 y 1,6 para valores $7-8$ y $>8$, vs $<7$ ), número de antidiabéticos orales prescritos (ADO) (OR:2,5, 4,1 y 5,7 para 1, 2 ó 3+ $\mathrm{ADO} v s$ no tratamiento farmacológico), tipo de ADO (mayor en sulfonilureas y glitazonas) y enfermedad respiratoria crónica (OR:0,5). Controlando el efecto de estos factores, el uso de AMGS se asoció adicionalmente a los años de ejercicio profesional del médico y a la asistencia en algunos departamentos sanitarios.

Conclusiones: La mitad de los pacientes con DM-NTI utilizan AMGS. Su uso se asoció fuertemente a las características clínicas de los pacientes, aunque algunos factores no clínicos explican parte de la variabilidad en su utilización.

Palabras clave: Diabetes Mellitus tipo 2. Automonitorización de glucosa en sangre. Utilización de medicamentos. Antidiabéticos orales. Estudios transversales.

\section{ABSTRACT}

\section{Prevalence and Associated Factors in}

\section{Correspondencia}

Salvador Peiró

Centro Superior de Investigación en Salud Pública (CSISP-FISABIO)

Avda Blasco Ibáñez, 17

46010 Valencia, España

Correo electrónico: peiro_bor@gva.es.

\section{Self-Monitoring of Blood Glucose in Noninsulin-treated Type 2 Diabetes Patients in the Valencia Community, Spain}

Background: Self-monitoring of blood glucose (SMBG) in noninsulintreated type 2 diabetes patients (T2DM) is a controversial topic.. We aimed to describe the SMBG prevalence in noninsulin-treated (NIT) T2DM patients in the Valencia Community (VC) and to analyze factors associated with their use.

Method: Cross-sectional study with 573 NIT-T2DM patients from 83 primary care surgeries. Electronic medical records and patient interview were used. We examine associations among patient, doctors and organizational characteristics, and the SMBG indication.

Results: $289(50.4 \%)$ of the 573 NIT-T2DM patients used SMBG. In the multivariable analysis SMBG use was associated with age (OR:2.3 for 65-84 and 6.0 for $<65$ years $v s$ 85+ years old)) the length from diagnosis (OR:2.2 for 10-15 years $v s$ 0-5 years), the number of OAAs prescribed (OR:2.5, 4.1 and 5.7 for 1,2 or $3+$ OAAs $v s$ no treatment with OAAs) and type (with more SMBG prescribed in patients with sulfonylureas and glitazones), glycated haemoglobin figures (OR: 1.9 y 1.6 for $7-8$ and $>8, v s<7$ ), sedentary behaviour (OR:1.6), obesity (OR:1.5), and housewife status, and chronic respiratory disease antecedents (OR:0.5). After controlling these factors, SMBG use was also associated with the length of doctor's professional practice and some healthcare departments.

Conclusions: In the Valencia Community the SMBG use in noninsulintreated T2DM patients is strongly associated with clinic patient' characteristics, but some non-clinical factors explain part of the variance in their utilization.

Keywords: Diabetes Mellitus, Type 2. Blood Glucose Self-Monitoring Drug utilization. Hypoglycemic Agents. Cross-Sectional Studies. Spain. 


\section{INTRODUCCIÓN}

La automonitorización de glucosa en sangre (AMGS) está ampliamente aceptada como una parte esencial del cuidado de las personas diabéticas tratadas con insulina, contribuyendo al ajuste de las dosis y la mejora de su control glucémico. Sin embargo, en el caso de la diabetes mellitus tipo 2 (DM2)no tratada con insulina (NTI) su valor es objeto de controversia ${ }^{1,2}$. Más allá de los argumentos sobre su posible utilidad para pacientes y médicos ${ }^{3,4}$, las evidencias empíricas muestran una escasa efectividad de la AMGS para mejorar el control glucémico en la DM2-NTI. Aunque los estudios muestran resultados contradictorios, la AMGS no ha mostrado reducciones significativas de los niveles de hemoglobina glicosilada (Hb1Ac) en diversos ensayos clínicos aleatorizados ${ }^{5-9}$ y diferentes revisiones sistemáticas y meta-análisis apoyan la nula o escasa eficacia de la AMGS para reducir los niveles de $\mathrm{Hb} 1 \mathrm{Ac}$, de modo que incluso cuando sus resultados son estadísticamente significativos, la magnitud del efecto es pequeña y su relevancia clínica discutible ${ }^{10-15}$.

Las evaluaciones económicas, consistentes con la escasa efectividad señalada en los estudios clínicos, muestran una baja relación coste-efectividad ${ }^{14,16-18} \mathrm{y}$, adicionalmente, algunos trabajos observacionales han mostrado una escasa adecuación de la indicación de AMGS en la práctica clínica real ${ }^{19-22}$. En este sentido, algunos estudios han cuantificado el ahorro que podría derivarse de restringir el uso de la AMGS a los grupos de diabéticos con una indicación adecuada a las recomendaciones de las guías de práctica clínica ${ }^{23,24}$. En el Sistema Nacional de Salud (SNS) español también se ha puesto en entredicho la efectividad ${ }^{20}$, eficiencia ${ }^{16} \mathrm{y}$ adecuación de la indicación de la AMGS en la práctica clínica real, hasta con un $65 \%$ de pacientes que reciben tiras de "utilización dudosamente indicada" 22 .
La incertidumbre sobre el valor substantivo de la AMGS para mejorar el control glucémico no ha impedido la rápida extensión de su uso entre las personas con DM2-NTI, lo que en todos los países desarrollados se ha traducido en un acelerado crecimiento del consumo de reactivos y consumibles y en el gasto asociado a los $\operatorname{mismos}^{25-31}$. Aunque no hemos hallado datos para el SNS, en la Comunidad Valenciana se realizaron 1.000 .000 de prescripciones en 2008 (dos tercios fueron para diabéticos no tratados con insulina) con un coste mensual en torno a los 4,5 millones de euros ${ }^{32}$ y en 2005 el $28 \%$ del gasto global destinado a la diabetes mellitus correspondió a material de autoanálisis (frente al $45 \%$ destinado a fármacos para tratar esta enfermedad ${ }^{33}$.

Pese a la preocupación por el crecimiento del gasto, la prevalencia y características de las personas diabéticas NTI que usan AMGS ha sido muy poco estudiada en nuestro entorno y la información sobre los factores clínicos y organizacionales asociados a la indicación de la AMGS en DM2-NTI es muy escasa.

Los objetivos de este trabajo son describir la prevalencia de la AMGS entre los pacientes con DM2-NTI en la Comunidad Valenciana, analizar los factores clínicos asociados a su indicación y valorar la importancia de posibles factores no clínicos que contribuyan a la variabilidad en su uso.

\section{SUJETOS Y MÉTODOS}

Diseño y ámbito. Estudio transversal realizado en las consultas de atención primaria $(n=83)$ de 10 Departamentos de Salud de la Comunidad Valenciana (Vinaròs, Castelló, La Plana, Sagunt, València-Clínic-La Malva-rosa, Xátiva-Ontinyent, Alcoi, Dénia, La Marina Baixa y Alacant-Sant Joan d'Alacant). 
Sujetos. Participaron en el estudio las personas de 30 y más años diagnosticadas de DM2 antes de 2009, que acudieron por cualquier motivo a las consultas de medicina general durante el periodo de selección de los participantes (marzo-abril 2010). Se emplearon los siguientes criterios de exclusión: 1) residir habitualmente en otro departamento de salud, comunidad autónoma o país, 2) embarazo, 3) indicación de AMGS por motivos diferentes a la DM2 y 4) no firmar el consentimiento informado permitiendo el acceso a la historia clínica para los fines del estudio. El tamaño muestral, asumiendo una prevalencia de DM2-NTI del $8 \%$ y un $50 \%$ de utilización de $\mathrm{AMGS}^{20}$, se estimó en 600 personas con DM2-NTI para una precisión del $4 \%$ y un nivel de confianza del $95 \%$.
Los investigadores seleccionaron aleatoriamente a 100 médicos generales (10 de cada Departamento de Salud participante) de los que 83 aceptaron participar. Estos médicos fueron formados en las variables y definiciones del estudio y recibieron un cuaderno de recogida de datos que incluía instrucciones y definiciones. Cada uno debía seleccionar a 10 pacientes con DM2 (el primer paciente del día que cumpliera los criterios de inclusión), obtener su consentimiento informado y registrar la información en el cuaderno de recogida de datos. Finalmente, se seleccionaron 823 personas con DM2 de las que se excluyeron 2 por ser menores de 30 años y 11 porque la variable principal del estudio, el uso de AMGS, no había sido cumplimentada (figura 1). De los 810 restantes $237(29,6 \%)$ estaban tratados con

Figura 1

\section{Diagrama del estudio}

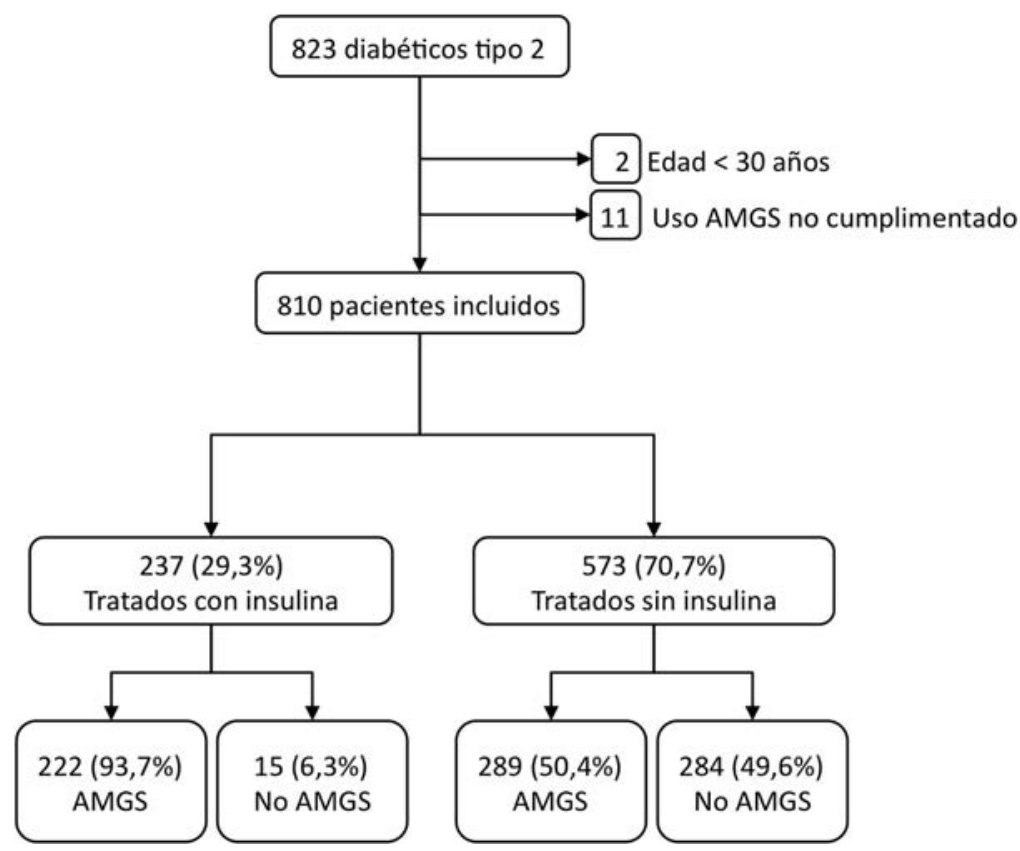


insulina y $573(70,7 \%)$ estaban tratados con antidiabéticos orales (ADO) o sin fármacos, siendo estos últimos la población objeto de estudio.

Fuentes de datos. Historia clínica electrónica y entrevista con el paciente. Cuando existía la posibilidad de diversas medidas en la historia clínica (por ejemplo, valores de presión arterial, glucemia, lípidos, etc.) se incluyó el valor de fecha más cercana a la entrevista siempre que se hallara en los 365 días previos a la misma (no se incluyeron valores previos a esta fecha).

Medida principal de resultado. Utilización de AMGS definida como tener registrada en la historia clínica informatizada la indicación de autocontrol glucémico por algún facultativo (excluyendo los pacientes que se monitorizan la glucemia por su cuenta sin indicación del médico).

Otras variables y definiciones. Sociodemográficas: edad (agrupada por tramos: $<55,55-64,65-74$ y 75 y más años), sexo, situación laboral (activo, activo parado, activo de baja por enfermedad, jubilado, ama de casa), cobertura farmacéutica (activo, pensionista), residencia (rural, urbana, urbanizaciones aisladas). Clínicas: años de evolución de la diabetes, factores de riesgo (hipertensión, dislipemia, tabaquismo, obesidad (IMC: $>30$ ) o sobrepeso (IMC: $>25$ y $<30$ ) y sedentarismo), antecedentes personales de enfermedad cardiovascular [cardiopatía isquémica, enfermedad vascular cerebral y enfermedad vascular periférica), asma o enfermedad pulmonar obstructiva crónica (EPOC)] y complicaciones de la diabetes (pie diabético, retinopatía, nefropatía). Farmacológicas: número de tiras reactivas/mes de glucosa pautadas en la historia clínica informatizada y consumo de tiras reactivas/mes declarado por el paciente, tratamiento actual de la DM2 (incluyendo los pacientes controlados sin fármacos). Utilización de servicios: frecuentación (visitas en los últimos 12 meses) al medico general, a enfermería de atención primaria, a atención continuada de atención primaria, a atención especializada (endocrinología, medicina interna) y frecuentación a urgencias o ingresos hospitalarias por hiper o hipoglucemia. De resultados: último valor de HbAlc y último valor de glucemia. Del médico general: edad, sexo, tipo centro (docente, no docente), años de ejercicio, número de pacientes asignados (total y con diagnóstico de DM2).

Ética y confidencialidad. El estudio, de naturaleza observacional y sin ningún tipo de intervención o pruebas derivadas del mismo, no implicaba riesgos adicionales para los pacientes. Todos los pacientes firmaron el correspondiente consentimiento informado previamente a su inclusión en el estudio para autorizar el acceso a su historia clínica. El cuaderno de recogida de datos no incluía ninguno identificativo de los pacientes ni de los facultativos participantes y la información personal quedaba anónima irreversiblemente cuando se remitía a los investigadores (esto explica la existencia de diversas variables con "datos perdidos" ya que no se pudo recuperar la información no incluida originalmente en el cuaderno de recogida de datos). Las bases de datos construidas para el análisis no incluyeron ningún identificador personal.

Análisis estadístico. En primer lugar, tras excluir a los pacientes tratados con insulina, se describieron las características sociodemográficas, clínicas y de tratamiento de los pacientes, las características disponibles de médicos y de las organización sanitaria, así como el porcentaje de pacientes con AMGS según cada una de estas características. Se analizó la fuerza de la asociación entre estas características y la prescripción de AMGS estimando las correspondientes odds ratio (OR) cruda con su intervalo de confianza del 95\% (IC95\%).

A continuación se analizaron las posibles asociaciones independientes entre las 
características de los pacientes y el uso del AMGS mediante un modelo de regresión logística multivariable. Se partió del modelo saturado y se utilizo una estrategia de exclusión-inclusión por pasos (backwardforward stepwise) con probabilidades de exclusión de 0,10 y de reinclusión de 0,05 para eliminar las variables no significativas. Se utilizó la opción con error estándar robusto [vee(cluster)] para controlar la autocorrelación residual entre pacientes tratados por los mismos médicos. La capacidad discriminativa del modelo se valoró mediante el C-Statistics y su calibración mediante la prueba de Hoshmer-Lemeshow. Con este modelo se estimó para cada paciente su probabilidad de tener indicada la AMGS en función de sus características socio-demográficas, clínicas y de tratamiento (propensity score).

Finalmente se desarrolló un segundo modelo de regresión logística incorporando las probabilidades esperadas del propensity score y las características de los médicos y la organización, para valorar el impacto de estas características sobre el uso de la AMGS controlando por las características clínicas de los pacientes. Para la construcción de este modelo y la valoración de su rendimiento se utilizó una estrategia de análisis similar a la del modelo de regresión logística previo. Todos los análisis se realizaron con el paquete estadístico STATA versión 9 (StataCorp, College Station, Texas).

\section{RESULTADOS}

De los 573 pacientes incluidos en el análisis $289(50,4 \%)$ tenían prescrita AMGS por sus médicos. En la tabla 1 se muestran las características de los pacientes incluidos y las asociaciones bivariables entre estas características y el uso de AMGS. El 61\% de los pacientes tenía 65 o más años, el $77 \%$ eran pensionistas y el $52 \%$ eran hombres. El 92\% tenía más de un factor de riesgo (fundamentalmente dislipemia e hipertensión arterial) y el $38 \%$ tenía alguna complicación (sobre todo cardiopatía isquémica y retinopatía). La menor edad (OR:1,8 y 3,5 para los pacientes con 64-74 y $<55$ años vs los de $75+$ años), la prestación con copago (OR:1,7), la situación laboral en activo (OR:1,7 vs jubilado) y la residencia en áreas urbanas (OR:1,8 vs núcleos aislados) se asociaron a una mayor utilización de AMGS en el análisis bivariable. Por el contrario, ninguno de los factores de riesgo ni la presencia de complicaciones o enfermedad respiratoria crónica se asoció al uso de AMGS.

Respecto al tratamiento (tabla 2), el 6\% de los pacientes con DM2-NTI no tenían tratamiento farmacológico, el 44\% estaba en tratamiento con ADO en monoterapia y un $50 \%$ con combinaciones de ADO. Las biguanidas fueron el ADO más utilizado $(75 \%)$, seguidas de las sulfonilureas (30\%) e incretinas (24\%). El 7\% utilizaba glitazonas. El uso de AMGS aumentó con la intensidad del tratamiento farmacológico (desde el $22 \%$ en los pacientes sin ningún ADO hasta el $73 \%$ en los pacientes con 3 ó más ADO), asociándose especialmente al uso de sulfonilureas, incretinas y glitazonas.

En la tabla 3 se muestran las características de los médicos, consultas y centros de salud participantes, así como las asociaciones bivariables entre estas características y la proporción de pacientes con DM2-NTI con prescripción de AMGS. Las consultas con mayor número de pacientes asignados (OR:1,5 para consultas con más de 1.500 pacientes $v s$ consultas con menos) y aquellas que no registraron el número de diabéticos (OR:2,6 vs consultas con 100 o menos diabéticos), así como la asignación a algunos Departamentos de Salud (OR:3,3 y 4,0 para los Departamentos I y J vs el Departamento A) se asociaron a una mayor utilización de la AMGS en este análisis bivariable. El 
Tabla 1

Utilización de automonitorización de la glucosa en sangre según características sociodemográficas y clínicas. Diabéticos tipo 2 no insulinizados. Análisis bivariable.

\begin{tabular}{|c|c|c|c|c|c|c|c|}
\hline & & $\mathrm{n}$ & $\%$ & $\begin{array}{c}\% \\
\text { AMGS }\end{array}$ & OR & IC95\% OR & $\mathrm{p}$ \\
\hline \multirow{4}{*}{ Edad } & $75+$ años & 147 & 25,79 & 38,10 & 1,00 & & 0,0003 \\
\hline & 65-74 años & 200 & 35,09 & 52,20 & 1,80 & $1,16-2,77$ & \\
\hline & 55-64 años & 151 & 26,49 & 52,32 & 1,78 & $1,12-2,83$ & \\
\hline & $<55$ años & 72 & 12,63 & 68,06 & 3,46 & $1,91-6,29$ & \\
\hline \multirow{2}{*}{ Sexo } & Mujer & 276 & 48,42 & 48,91 & 1,00 & & 0,4554 \\
\hline & Hombre & 294 & 51,58 & 52,04 & 1,13 & $0,82-1,57$ & \\
\hline \multirow{2}{*}{$\begin{array}{l}\text { Prestación } \\
\text { farmacéutica }\end{array}$} & "Pensionista" & 434 & 76,68 & 47,47 & 1,00 & & 0,0080 \\
\hline & "Activo" & 132 & 23,32 & 60,61 & 1,70 & $1,15-2,53$ & \\
\hline \multirow{3}{*}{$\begin{array}{l}\text { Situación } \\
\text { laboral }\end{array}$} & Jubilado & 329 & 58,02 & 45,90 & 1,00 & & 0,0361 \\
\hline & Ama de casa & 120 & 21,16 & 52,50 & 1,30 & $0,86-1,98$ & \\
\hline & En activo & 118 & 20,81 & 59,32 & 1,72 & $1,12-2,63$ & \\
\hline \multirow{3}{*}{ Residencia } & Núcleo aislado & 236 & 42,22 & 41,95 & 1,00 & & $\overline{0,0024}$ \\
\hline & Núcleo urbano & 312 & 55,81 & 56,41 & 1,79 & $1,27-2,52$ & \\
\hline & Urbanizaciones & 11 & 1,97 & 50,45 & 2,42 & $0,69-8,50$ & \\
\hline \multirow{4}{*}{$\begin{array}{l}\text { Años de } \\
\text { evolución } \\
\text { de la diabetes }\end{array}$} & $<5$ & 260 & 45,38 & 43,46 & 1,00 & & 0,0084 \\
\hline & $5 \mathrm{a}<10$ & 152 & 26,53 & 51,97 & 1,41 & $0,94-2,10$ & \\
\hline & 10 a 15 & 87 & 15,18 & 62,07 & 2,13 & $1,29-3,50$ & \\
\hline & $15+$ & 74 & 12,91 & 58,11 & 1,80 & $1,07-3,04$ & \\
\hline \multirow{3}{*}{ Obesidad } & Normal & 195 & 34,39 & 46,67 & 1,00 & & 0,2612 \\
\hline & Sobrepeso & 209 & 36,86 & 49,28 & 1,11 & $0,75-1,64$ & \\
\hline & Obesidad & 163 & 28,75 & 52,21 & 1,41 & $0,93-2,14$ & \\
\hline \multirow{2}{*}{ Tabaquismo } & No fumador & 498 & 87,37 & 49,60 & 1,00 & & 0,2431 \\
\hline & Fumador & 72 & 12,63 & 56,94 & 1,34 & $0,82-2,21$ & \\
\hline \multirow{2}{*}{$\begin{array}{l}\text { Actividad } \\
\text { Física }\end{array}$} & Regular & 364 & 64,77 & 47,80 & 1,00 & & 0,1265 \\
\hline & Nunca/ocasional & 198 & 35,23 & 54,55 & 1,31 & $0,93-1,85$ & \\
\hline \multirow{2}{*}{ Dislipemia } & No & 163 & 28,65 & 52,15 & 1,00 & & 0,6432 \\
\hline & $\mathrm{Si}$ & 406 & 71,35 & 50,00 & 0,92 & $0,64-1,32$ & \\
\hline \multirow{2}{*}{$\begin{array}{l}\text { Hipertensión } \\
\text { arterial }\end{array}$} & No & 108 & 19,15 & 58,33 & 1,00 & & 0,0589 \\
\hline & $\mathrm{Si}$ & 456 & 80,85 & 48,25 & 0,67 & $0,44-1,01$ & \\
\hline \multirow{4}{*}{$\begin{array}{l}\text { Sumatorio } \\
\text { factores } \\
\text { de riesgo* }\end{array}$} & $0 / 1$ & 54 & 9,87 & 50,00 & 1,00 & & 0,5450 \\
\hline & 2 & 181 & 33,09 & 47,51 & 0,91 & $0,49-1,66$ & \\
\hline & 3 & 209 & 38,21 & 49,28 & 0,97 & $0,53-1,77$ & \\
\hline & $4 / 5$ & 103 & 18,83 & 56,31 & 1,29 & $0,67-2,49$ & \\
\hline \multirow{2}{*}{ Retinopatía } & No & 491 & 85,69 & 51,73 & 1,00 & & 0.1288 \\
\hline & $\mathrm{Si}$ & 82 & 14,31 & 42,68 & 0,69 & $0,43-1,11$ & \\
\hline \multirow{2}{*}{ Nefropatía } & No & 510 & 89,01 & 50,98 & 1,00 & & 0.4584 \\
\hline & $\mathrm{Si}$ & 63 & 10,99 & 46,03 & 0,82 & $0,48-1,39$ & \\
\hline \multirow{2}{*}{$\begin{array}{l}\text { Cardiopatía } \\
\text { Isquémica }\end{array}$} & No & 489 & 85,34 & 49,69 & 1,00 & & 0,3904 \\
\hline & $\mathrm{Si}$ & 84 & 14,66 & 54,76 & 1,23 & $0,77-1,95$ & \\
\hline \multirow{2}{*}{$\begin{array}{l}\text { Enf. Vascular } \\
\text { cerebral }\end{array}$} & No & 545 & 95,11 & 50,46 & 1,00 & & \\
\hline & $\mathrm{Si}$ & 28 & 4,89 & 50,00 & 0,98 & $0,46-2,10$ & 0,9622 \\
\hline
\end{tabular}




\section{Tabla 1}

Utilización de automonitorización de la glucosa en sangre según características socio-demográficas y clínicas. Diabéticos tipo 2 no insulinizados. Análisis bivariable. (Continuación)

\begin{tabular}{|l|c|c|c|c|c|c|c|}
\hline & & $\mathrm{n}$ & $\%$ & $\begin{array}{c}\% \\
\text { AMGS }\end{array}$ & OR & IC95\% OR & $\mathrm{p}$ \\
\hline \multirow{2}{*}{$\begin{array}{l}\text { Enf. vascular } \\
\text { Periférica }\end{array}$} & $\mathrm{No}$ & 528 & 92,15 & 50,00 & 1,00 & & \\
\hline \multirow{2}{*}{ Pie diabético } & $\mathrm{Si}$ & 45 & 7,85 & 55,56 & 1,25 & $0,68-2,30$ & 0,4738 \\
\cline { 2 - 8 } & $\mathrm{No}$ & 559 & 97,56 & 50,09 & 1,00 & & \\
\hline \multirow{2}{*}{ Asma/EPOC } & $\mathrm{Si}$ & 14 & 2,44 & 64,29 & 1,79 & $0,59-5,42$ & 0,2905 \\
\hline & $\mathrm{No}$ & 517 & 90,23 & 51,26 & 1,00 & & \\
\hline \multirow{2}{*}{$\begin{array}{l}\text { Cumatorio } \\
\text { Comorbilidad }\end{array}$} & $\mathrm{Si}$ & 56 & 9,77 & 42,86 & 0,71 & $0,41-1,24$ & 0,2318 \\
\cline { 2 - 8 } & 0 & 360 & 62,83 & 49,44 & 1,00 & & 0,5583 \\
\hline TOTAL & 1 & 138 & 24,08 & 54,35 & 1,22 & $0,82-1,80$ & \\
\hline
\end{tabular}

Datos perdidos: edad (3), sexo (3), prestación farmacéutica (7), situación laboral (6), residencia (14); obesidad (6), tabaquismo (3), actividad física (11), dislipemia (4), hipertensión (9). *Factores de riesgo: obesidad/sobrepeso, tabaquismo, sedentarismo, dislipemia e hipertensión; **Complicaciones o comorbilidad: nefropatía, cardiopatía isquémica, ictus, enfermedad vascular periférica, pie diabético y EPOC. AMGS: automonitorización glucosa en sangre; OR: Odds ratio; IC95: Intervalo de confianza del 95\%. EPOC: enfermedad pulmonar obstructiva crónica.

Tabla 2

Utilización de automonitorización de la glucosa en sangre según tratamiento. Diabéticos tipo 2 no insulinizados. Análisis bivariable

\begin{tabular}{|c|c|c|c|c|c|c|c|}
\hline & & $\mathrm{n}$ & $\%$ & $\%$ AMGS & OR & IC95\% OR & $\mathrm{p}$ \\
\hline \multirow{2}{*}{$\begin{array}{l}\text { Tratamiento } \\
\text { diabetes }\end{array}$} & No farmacológico & 36 & 6,28 & 22,22 & 1,00 & & 0,0003 \\
\hline & Farmacológico & 537 & 93,72 & 52,33 & 3,84 & $1,72-8,58$ & \\
\hline \multirow{2}{*}{ Sulfonilureas } & No & 403 & 70,33 & 45,41 & 1,00 & & 0,0002 \\
\hline & $\mathrm{Si}$ & 170 & 29,67 & 62,35 & 1,99 & $1,38-2,87$ & \\
\hline \multirow{2}{*}{ Biguanidas } & No & 146 & 25,48 & 45,89 & 1,00 & & 0,2030 \\
\hline & $\mathrm{Si}$ & 427 & 74,52 & 51,99 & 1,27 & $0,88-1,86$ & \\
\hline \multirow{2}{*}{ Meglitinidas } & No & 484 & 84,47 & 50,21 & 1,00 & & 0,7976 \\
\hline & $\mathrm{Si}$ & 89 & 15,53 & 51,69 & 1,06 & $0,67-1,67$ & \\
\hline \multirow{2}{*}{ Glitazonas } & No & 532 & 92,84 & 48,68 & 1,00 & & 0,0021 \\
\hline & $\mathrm{Si}$ & 41 & 7,16 & 73,17 & 2,87 & $1,41-5,86$ & \\
\hline \multirow{2}{*}{ Incretinas } & No & 436 & 76,09 & 46,56 & 1,00 & & 0,0009 \\
\hline & $\overline{\mathrm{Si}}$ & 137 & 23,91 & 62,77 & 1,94 & $1,30-2,87$ & \\
\hline \multirow{4}{*}{ Combinaciones } & No tratados & 37 & 6,46 & 24,32 & 1,00 & & $<0,0001$ \\
\hline & ADO único & 251 & 43,80 & 41,83 & 2,24 & $1,01-4,94$ & \\
\hline & $\mathrm{ADO}(2)$ & 226 & 39,44 & 58,41 & 4,37 & $1,97-9,69$ & \\
\hline & $\mathrm{ADO}(3+)$ & 59 & 10,30 & 72,88 & 8,36 & $3,24-21,52$ & \\
\hline TOTAL & & 573 & 100,00 & 50,44 & & & \\
\hline
\end{tabular}

AMGS: autocontrol glucémico; OR: Odds ratio; IC95: Intervalo de confianza del 95\%. ADO: Antidiabéticos orales 
Tabla 3

Utilización de automonitorización de la glucosa en sangre según características de los médicos, cupos y centros de salud. Diabéticos tipo 2 no insulinizados. Análisis bivariable

\begin{tabular}{|c|c|c|c|c|c|c|c|}
\hline & & $\mathrm{n}$ & $\%$ & \%AMGS & OR & IC95\% OR & $\mathrm{p}$ \\
\hline \multirow{3}{*}{ Edad médico } & $<45$ años & 130 & 22,69 & 53,08 & 1,00 & & \\
\hline & $45-54$ años & 301 & 52,53 & 53,49 & 1,02 & $0,67-1,53$ & 0,937 \\
\hline & $\overline{55+\text { años }}$ & 142 & 24,78 & 41,55 & 0,63 & $0,39-1,01$ & 0,058 \\
\hline \multirow{2}{*}{ Sexo médico } & Hombre & 289 & 50,44 & 47,40 & 1,00 & & \\
\hline & Mujer & 284 & 49,56 & 52,60 & 1,28 & $0,92-1,77$ & 0,143 \\
\hline \multirow{4}{*}{ Años ejercicio } & $0-15$ años & 99 & 17,28 & 52,53 & 1,00 & & \\
\hline & $16-25$ años & 246 & 42,93 & 51,22 & 0,95 & $0,59-1,51$ & 0,826 \\
\hline & $>25$ años & 167 & 29,14 & 47,31 & 0,81 & $0,49-1,33$ & 0,411 \\
\hline & $\mathrm{ns} / \mathrm{nc}$ & 61 & 10,65 & 52,46 & 0,99 & $0,53-1,89$ & 0,993 \\
\hline \multirow{3}{*}{$\begin{array}{l}\text { Pacientes } \\
\text { asignados }\end{array}$} & $<=1500$ pacientes & 216 & 37,70 & 43,52 & 1,00 & & \\
\hline & $>1500$ pacientes & 326 & 56,89 & 54,29 & 1,54 & $1,09-2,18$ & 0,014 \\
\hline & $\mathrm{ns} / \mathrm{nc}$ & 31 & 5,41 & 58,06 & 1,79 & $0,84-3,85$ & 0,132 \\
\hline \multirow{3}{*}{$\begin{array}{l}\text { Diabéticos } \\
\text { asignados }\end{array}$} & $<=100$ diabéticos & 174 & 30,37 & 41,95 & 1,00 & & \\
\hline & $>100$ diabéticos & 303 & 52,88 & 50,50 & 1,41 & $0,97-2,06$ & 0,073 \\
\hline & $\mathrm{ns} / \mathrm{nc}$ & 96 & 16,75 & 65,62 & 2,64 & $1,57-4,43$ & $<0,001$ \\
\hline \multirow{2}{*}{$\begin{array}{l}\begin{array}{l}\text { Centro } \\
\text { docente }\end{array} \\
\end{array}$} & $\mathrm{Si}$ & 219 & 38,22 & 48,40 & 1,00 & & \\
\hline & No & 354 & 61,78 & 51,69 & 1,14 & $0,81-1,60$ & 0,444 \\
\hline \multirow{10}{*}{$\begin{array}{l}\text { Departamento } \\
\text { de salud }\end{array}$} & $\overline{\mathrm{A}}$ & 25 & 4,36 & 36,00 & 1,00 & & \\
\hline & $\bar{B}$ & 67 & 11,69 & 38,81 & 1,13 & $0,43-2,92$ & 0,805 \\
\hline & $\mathrm{C}$ & 54 & 9,42 & 38,89 & 1,13 & $0,42-3,02$ & 0,806 \\
\hline & $\bar{D}$ & 79 & 13,79 & 39,24 & 1,15 & $0,45-2,92$ & \begin{tabular}{|l|}
0,772 \\
\end{tabular} \\
\hline & $E$ & 73 & 12,74 & 47,95 & 1,64 & $0,64-4,18$ & 0,302 \\
\hline & $F$ & 61 & 10,65 & 50,82 & 1,84 & $0,70-4,79$ & 0,214 \\
\hline & $\bar{G}$ & 41 & 7,16 & 53,66 & 2,06 & $0,74-5,72$ & 0,166 \\
\hline & $\mathrm{H}$ & 38 & 6,63 & 60,53 & 2,73 & $0,96-7,74$ & 0,060 \\
\hline & $\mathrm{I}$ & 63 & 10,99 & 65,08 & 3,31 & $1,26-8,71$ & 0,015 \\
\hline & $\mathrm{J}$ & 72 & 12,57 & 69,44 & 4,04 & $1,55-10,53$ & 0,004 \\
\hline $\begin{array}{l}\text { TOTAL } \\
\end{array}$ & & 573 & 100,00 & 50,44 & & & \\
\hline
\end{tabular}

años ejercicio (89), tamaño cupo (40), número diabéticos (133) ACG: autocontrol glucémico; OR: Odds ratio; IC95\%: Intervalo de confianza del 95\%. AO: Antidiabéticos orales.

resto de variables no mostraron asociaciones estadísticamente significativas con el uso de AMGS, aunque algunas como la edad del médico (menor uso de AMGS a mayor edad) o el número de diabéticos asignados a la consulta (mayor uso en consultas con más de 100 diabéticos) rozaron la significación estadística.

En la tabla 4 se muestran las características de los pacientes que se asociaron independientemente al uso del AMGS.
Estas incluyeron: la menor edad (OR:2,3 para 65-84 años y 6,0 para $<65$ años, $v s$ $85+$ años), los años de evolución de la diabetes (OR:2,2 para 10-15 años vs 0-5 años), la intensidad del tratamiento con ADO (OR:2,5, 4,1 y 5,7 para 1, 2 ó 3+ ADO vs no tratamiento farmacológico), algunos tratamientos específicos (sulfonilureas, glitazonas), los niveles de hemoglobina glicosilada (OR:1,9 y 1,6 para valores 7-8 y $>8, v s<7$ ), algunos factores de riesgo como el sedentarismo (OR: 1,6$)$ 
Tabla 4

Factores del paciente asociados a la utilización del autocontrol glucémico. Diabéticos tipo 2 no insulinizados. Análisis de regresión logística múltiple

\begin{tabular}{|c|c|c|c|c|}
\hline & & OR & IC95\% OR & $\mathrm{p}$ \\
\hline \multirow{4}{*}{ Edad } & $85+$ años & 1,00 & & \\
\hline & 75-84 años & 2,26 & $1,36-3,78$ & 0,002 \\
\hline & 65-74 años & 2,31 & $1,31-4,08$ & 0,004 \\
\hline & $<65$ años & 6,02 & $2,96-12,24$ & $<0,001$ \\
\hline \multirow{2}{*}{ Situación laboral } & jubilado & 1,00 & & \\
\hline & ama de casa & 1,58 & $0,97-2,56$ & 0,066 \\
\hline \multirow{3}{*}{$\begin{array}{l}\text { Años evolución } \\
\text { diabetes }\end{array}$} & $0-5$ años & 1,00 & & \\
\hline & $10-15$ años & 2,22 & $1,23-3,99$ & 0,008 \\
\hline & $15+$ años & 1,73 & $0,94-3,21$ & 0,081 \\
\hline \multirow{2}{*}{ Actividad física } & Activo & 1,00 & & \\
\hline & Sedentarismo & 1,62 & $1,07-2,44$ & 0,022 \\
\hline \multirow{2}{*}{ Obesidad } & No & 1,00 & & \\
\hline & Sobrepeso/obeso & 1,54 & $1,01-2,34$ & 0,045 \\
\hline \multirow{2}{*}{ Asma/EPOC } & No & 1,00 & & \\
\hline & $\mathrm{Si}$ & 0,54 & $0,28-1,04$ & 0,066 \\
\hline \multirow{3}{*}{$\mathrm{Hb} 1 \mathrm{Ac}$} & $<7$ & 1,00 & & \\
\hline & $7-8$ & 1,91 & $1,21-3,00$ & 0,005 \\
\hline & $>8$ & 1,58 & $0,84-2,97$ & 0,160 \\
\hline \multirow{4}{*}{ Tratamiento } & No fármacos & 1,00 & & \\
\hline & ADO único & 2,47 & $0,99-6,13$ & 0,052 \\
\hline & $\mathrm{ADO}(2)$ & 4,08 & $1,59-10,47$ & 0,003 \\
\hline & $\overline{\mathrm{ADO}(3+)}$ & 5,74 & $1,77-18,63$ & 0,004 \\
\hline \multirow{2}{*}{ Sulfonilureas } & No & 1,00 & & \\
\hline & $\mathrm{Si}$ & 1,44 & $0,91-2,27$ & 0,117 \\
\hline \multirow{2}{*}{ Glitazonas } & No & 1,00 & & \\
\hline & $\mathrm{Si}$ & 2,08 & $0,82-5,29$ & 0,124 \\
\hline
\end{tabular}

$\mathrm{n}=511 ; \mathrm{p}<0,0001 ; \mathrm{r} 2: 0,130$; Log likelihood: $-308,144$; C-Statistic: 0,733; $\mathrm{p}\left(\chi^{2}\right)$ Hosmer-Lemeshow: 0,918. Variables no aceptadas por el modelo: sexo, copago, tabaquismo, hiperlipidemia, hipertensión, número de factores de riesgo, nefropatía, enfermedad vascular cerebral, retinopatía, pie diabético, enfermedad vascular periférica, cardiopatía isquémica, número de complicaciones, incretinas. OR: Odds ratio; IC95: Intervalo de confianza del 95\%. ADO: Antidiabéticos orales; EPOC: Enfermedad pulmonar obstructiva crónica.

o la obesidad (OR: 1,5) y la situación laboral "ama de casa" (OR:1,6 vs jubilado). Los antecedentes personales de asma o EPOC se asociaron a una reducción en la probabilidad de usar AMGS (OR:0,5). El modelo mostró una buena capacidad discriminativa $(\mathrm{C}$-Statistics $=0,73)$ y una excelente calibración [ $\mathrm{p}\left(\chi^{2}\right)$ HoshmerLemeshow: 0,918].
En la tabla 5 se muestran las características de médicos, consultas y centros que se asociaron de forma independiente al uso de AMGS tras controlar el efecto de las características de los pacientes que se relacionaban con su indicación identificadas en la tabla 4. Los años de ejercicio profesional (un 56\% más de uso de AMSG en los médicos con 16 a 25 años de ejercicio profesional respecto a los de 15 o menos 
Tabla 5

Factores de la organización sanitaria asociados a la utilización del autocontrol glucémico (controlando el efecto de las características clínicas).

Diabéticos tipo 2 no insulinizados. Análisis de regresión logística múltiple

\begin{tabular}{|l|c|c|c|c|}
\hline & & OR & IC95\% OR & $\mathrm{p}$ \\
\hline \multirow{2}{*}{$\begin{array}{l}\text { Años de ejercicio } \\
\text { profesional }\end{array}$} & $0-15$ años & 1,00 & & \\
\cline { 2 - 5 } & $16-25$ años & 1,58 & $1,06-2,37$ & 0,025 \\
\hline \multirow{3}{*}{$\begin{array}{l}\text { Departamento } \\
\text { de salud }\end{array}$} & $\mathrm{A}$ & 1,00 & & \\
\cline { 2 - 5 } & $\mathrm{H}$ & 2,34 & $1,06-5,21$ & 0,036 \\
\cline { 2 - 5 } & $\mathrm{I}$ & 2,32 & $1,21-4,44$ & 0,011 \\
\cline { 2 - 5 } & $\mathrm{J}$ & 3,67 & $1,98-6,80$ & $<0,001$ \\
\hline \multicolumn{2}{|l|}{ Probabilidad paciente por cada 0,10} & 1,59 & $1,42-1,77$ & $<0,001$ \\
\hline
\end{tabular}

$\mathrm{n}=511 ; \mathrm{p}<0,0001 ; \mathrm{r} 2: 0,168$; Log likelihood: -294.826; C-Statistic: 0,764; $\mathrm{p}\left(\chi^{2}\right)$ Hosmer-Lemeshow: 0,525. Variables no aceptadas por el modelo: edad del médico, sexo del médico, número de pacientes asignados, número de diabéticos asignados, acreditación docente. OR: Odds ratio; IC95: Intervalo de confianza del 95\%. años, controlando por la probabilidad esperada de llevar AMGS derivada de las características clínicas del paciente) y 3 Departamentos de Salud se asociaron al uso de AMSG. El modelo incrementó discretamente su capacidad discriminativa respecto al previo (C-Statistisc: 0,76$)$ y mantuvo la buena calibración $\left(\mathrm{p}\left(\chi^{2}\right)\right.$ Hoshmer-Lemeshow): 0,525). En la figura 2 se muestra la proporción de AMSG esperada en cada Departamento de Salud controlando la diversidad de las características clínicas de los pacientes tratados (probabilidad esperada según el modelo de la tabla 4) y la proporción realmente observada. Pese a los amplios intervalos de confianza, el Departamento A tuvo una proporción observada estadísticamente significativa inferior a la esperada, mientras que en el Departamento J esta proporción fue superior a la esperada.

Figura 2

Proporción de personas diabéticas tipo 2 no tratadas con insulina y con indicación de AMSG observada en cada Departamento de Salud, y proporción esperada en función de las características cínicas de sus pacientes

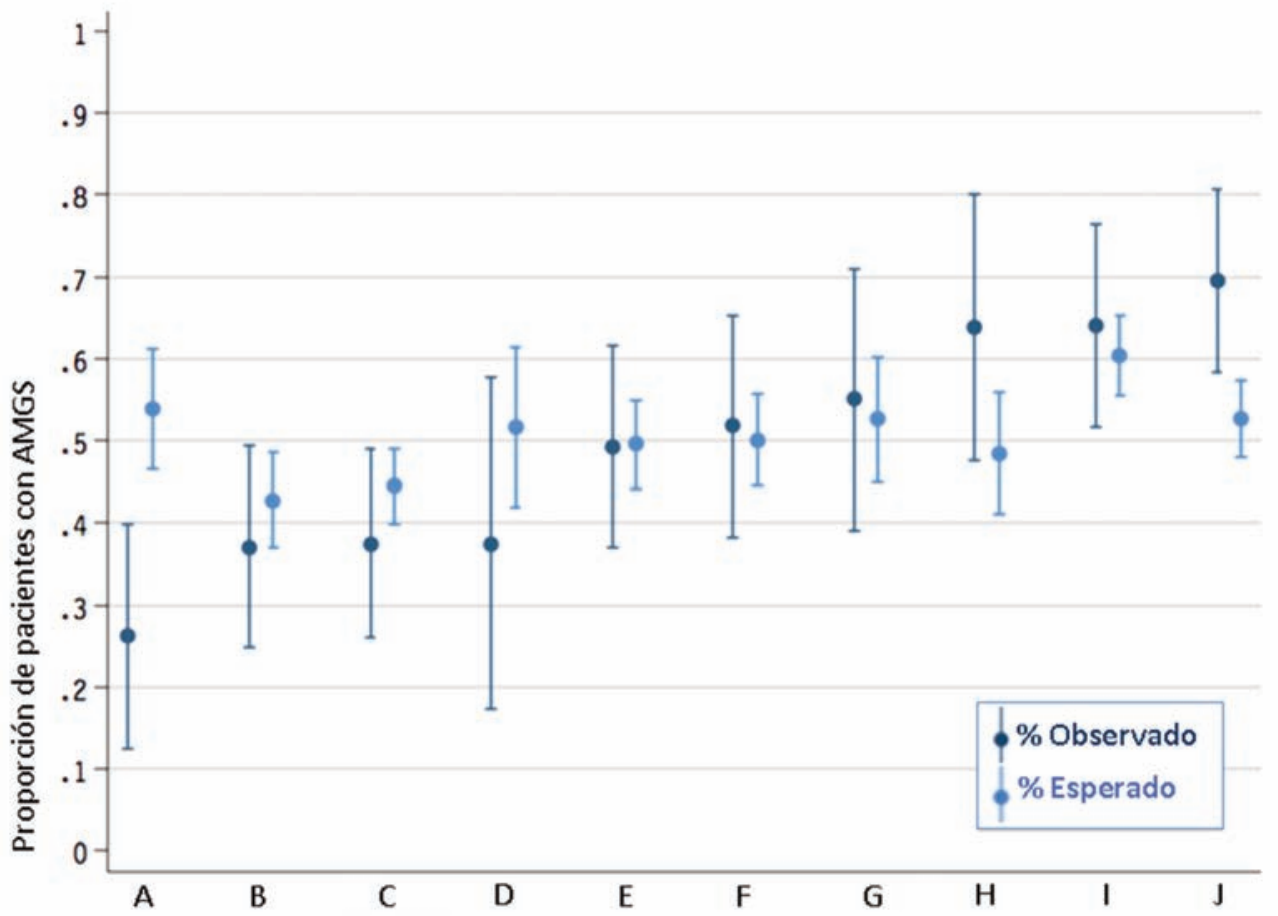

Departamentos de salud 


\section{DISCUSIÓN}

Los resultados de este estudio muestran: 1) que el porcentaje de uso de la AMGS en la Comunidad Valenciana en 2010 se situaba en el 50\% para los pacientes con DM2-NTI, 52\% para los tratados con $\mathrm{ADO}$ y $22 \%$ para los que no llevan tratamiento farmacológico; 2) que determinados factores clínicos, fundamentalmente la menor edad del paciente, la mayor duración de la enfermedad, los niveles de $\mathrm{Hb} 1 \mathrm{Ac}$ superiores a 7, el tipo e intensidad del tratamiento antidiabético y algunos factores de riesgo, se asocian fuertemente a la indicación de AMGS; 3 ) que algunos factores no clínicos, como los años de ejercicio profesional del médico que los atiende y el Departamento de Salud al que están asignados, también se asocian al uso de AMGS una vez controladas las diferencias en las características clínicas de los pacientes.

Respecto a la prevalencia de uso de AMGS, diversos estudios han mostrado una gran variabilidad en su utilización, tanto entre países ${ }^{34}$ como dentro de un mismo país ${ }^{25,35}$, variando incluso la prevalencia en el tipo de pacientes que reciben AMGS. Así, para 2007, algunos países como Alemania u Holanda parecen mantener un patrón de alto uso de la AMGS en pacientes insulinizados (Alemania: 90\%; Holanda: 95\%) pero bajo en tratados con ADO (Alemania: 35\%; Holanda: $36 \%$ ) y mínimo en los no tratados farmacológicamente (Alemania: 7\%; Holanda: $17 \%$ ), mientras que otros, como el Reino Unido, Australia o Noruega, muestran cifras elevadas en todos los grupos (Reino Unido: 93\%, 73\%, 54\%; Australia: $82 \%, 70 \%, 63 \%$; Noruega: $96 \%, 73 \%, 45 \%$, para personas diabéticas tratadas con insulina, con ADO y no tratadas, respectivamente $)^{34}$. El patrón hallado en la Comunidad Valenciana se situaría entre ambos grupos de países, manteniendo tasas altas de AMGS para las personas diabéticas tratadas con insulina (94\%), pero intermedias en los tratadas con $\mathrm{ADO}(50 \%)$ o no tratadas con fármacos $(22 \%)$. Esta variabilidad entre países puede estar relacionada con la variabilidad en las guías de práctica señalada en un trabajo que comparaba las de 13 países $^{36}$ y la incertidumbre y controversia sobre la efectividad de la AMGS.

Respecto a las variaciones en el mismo país, un estudio en 262 consultas de Reino Unido (1993-1998) reportó porcentajes entre el $12 \%$ y el $100 \%$ de diabéticos con AMGS según consultas ${ }^{25}$, y un estudio posterior (2003) en 300 trusts del Reino Unido mostró tasas entre 30 y 180 prescripciones de tiras reactivas para AMGS por 1.000 habitantes ${ }^{35}$. Nuestro trabajo también muestra una importante variabilidad entre Departamentos de Salud en el uso de la AMGS en DM2 no tratada con insulina (desde el 36\% al $69 \%$, sin ajustar por las características de los pacientes) aunque, dado el escaso tamaño muestral, las diferencias sólo son estadísticamente significativas en los extremos. Las importantes diferencias entre las guías de práctica clínica de las sociedades científicas españolas ${ }^{37}$ y la diversidad de políticas de las administraciones sanitarias de las Comunidades Autónomas (que pueden ir desde estimular su uso a intentar a reducirlo, con más o menos matices según grupos de pacientes) pueden ser factores locales que contribuyan a esta variabilidad.

En cuanto a los factores asociados al uso de la AMGS en pacientes con DM2-NTI, nuestros resultados son parcialmente coincidentes con los de los escasos estudios sobre el tema, aunque las comparaciones vienen dificultadas porque los diferentes estudios utilizan diferentes variables $y$, adicionalmente, diferentes definiciones. Entre las variables que son consistentes con otros estudios cabe destacar la intensidad del tratamiento, entendida como número de ADO que se utiliza un mismo paciente ${ }^{38-41}$, la mayor edad del paciente ${ }^{38,39,42,43}$, los valores de $\mathrm{Hb} 1 \mathrm{Ac}^{38}$ y el tiempo de duración de la diabetes $^{40}$. Otras variables del paciente que han mostrado asociación con el uso de 
AMGS en algunos estudios incluyen el mayor nivel educativo ${ }^{38}$, haber realizado más visitas médicas ${ }^{38}$, ser de grupos étnicos minoritarios ${ }^{42,44}$, la asistencia a programas de educación diabetológica ${ }^{40}$, el estar casado o con pareja estable ${ }^{40}$ (una asociación que, posiblemente, se relaciona con la hallada en nuestro estudio con la variable "amas de casa") y el haber reportado episodios de hipoglucemia ${ }^{40}$.

La incorporación de variables del médico y del Departamento de Salud a los factores explicativos del uso de AMGS sugieren que los factores no clínicos también influyen en la indicación de AMGS, aunque su efecto no es tan importante como el de los factores del paciente, tanto en nuestro estudio como en otros trabajos ${ }^{45}$. La asociación entre el mayor tiempo de ejercicio profesional y el mayor uso de AMGS puede tener varias explicaciones a nivel individual (mayor experiencia, conocimientos menos actualizados, u otros) u organizativo (ubicación en zonas urbanas, más jóvenes, con mayor número de pacientes asignados, mayor interacción con los especialistas u otras). El menor o mayor uso en algunos Departamentos de Salud puede tener relación con el mayor o menor uso de la AMGS por los especialistas de los correspondientes Departamentos o con el mayor o menor desarrollo de los programas de enfermería de educación diabetológica. El impacto de estos dos factores organizativos, especialistas en endocrinología y programas de enfermería ha sido muy poco estudiado, pese a su potencial para modificar las tasas de uso de la AMGS.

Este estudio tiene diversas limitaciones. En primer lugar, sólo incluye 10 de los 24 Departamentos de Salud de la Comunidad Valenciana y, por tanto, su representatividad es limitada. En segundo lugar, la muestra utilizada corresponde a la población que acude a consulta de atención primaria, no a la población general de la Comunidad Valenciana. Este tipo de muestras tiene diversos sesgos (mayor accesibilidad de las personas que no están laboralmente en activo, exclusión exclusión de la mayor parte de los funcionarios o de las personas tratadas en el sector privado, exclusión de pacientes con dificultades para desplazarse y otras) y, en este sentido, los resultados del estudio no son necesariamente generalizables al conjunto de la población. En tercer lugar, el muestreo sistemático utilizado permite ciertos sesgos de selección (por ejemplo, selección de pacientes de mayor nivel educativo con los que es más fácil desarrollar las entrevistas) que, aunque se intentaron minimizar mediante la formación de los médicos participantes, no pueden ser descartados.

En otro terreno, algunas variables que pueden asociarse al uso de la AMGS no fueron estudiadas. A nivel del paciente cabe destacar la ausencia de información sobre nivel educativo, nivel de renta, presencia de episodios de hiper o hipoglucemia en la evolución clínica o la asistencia a programas de educación diabetológica. Se trata de variables que se pueden asociar al uso de la AMGS y, también, actuar como factores de confusión con otras variables. Finalmente, nuestro estudio, de naturaleza transversal, no permite valorar las posibles relaciones causales entre el uso de AMGS y resultados clínicos como los niveles de $\mathrm{Hb} 1 \mathrm{Ac}$.

Aunque la incertidumbre sobre la efectividad de la AMGS en la DM2 no tratada con insulina convierte en objeto de controversia cualquier posible recomendación sobre su uso, las de la Sociedad Valenciana de Medicina Familiar y Comunitaria ${ }^{46}$ y las de la Agencia Valenciana de Salud ${ }^{32}$ no los consideran adecuado salvo en situaciones con riesgo de desestabilización. Desde esta posición, las cifras halladas en nuestro estudio sugieren una clara sobreutilización de la AMGS en nuestro entorno. Dado el elevado consumo de recursos que conlleva (que podrían ser utilizados de forma más efectiva para mejorar otros aspectos de la aten- 
ción a la diabetes u otras enfermedades) parece lógico desarrollar actuaciones que reconduzcan el uso de la AMGS hacia los pacientes en que sea más efectiva. La identificación de las características de los pacientes y de la organización sanitaria asociadas a la indicación de AMGS pueden ser de interés para desarrollar algunas estrategias con esta finalidad.

\section{AGRADECIMIENTOS}

A los médicos colaboradores en la recogida de la información de los 10 Departamentos de Salud de la Agencia Valenciana de Salud participantes. A Mercedes Jiménez Heredia, del Servicio de Farmacia del Hospital Clínic Universitari de Valencia, por su colaboración en el diseño y gestión de la base de datos. Este estudio fue promovido por la Sociedad Valenciana de Farmacéuticos de Atención Primaria.

\section{BIBLIOGRAFÍA}

1. Davison MB. Counterpoint: Self-monitoring of blood glucose in type 2 diabetic patients not receiving insulin: A waste of money. Diabetes Care. 2005;28:1531-3

2. Kennedy L. Self-monitoring of blood glucose in type 2 diabetes: time for evidence of efficacy. Diabetes Care. 2001;24:977-8.

3. Austin MM, Haas L, Johnson T, Parkin CG, Parkin CL, Spollett G, et al. Self-monitoring of blood glucose: benefits and utilization. Diabetes Educ. 2006;32:835$6,844-7$.

4. Banerji MA. The foundation of diabetes self-management: glucose monitoring. Diabetes Educ. 2007;33 Suppl 4:87S-90S.

5. Johnson JA, Majumdar SR, Bowker SL, Toth EL, Edwards A. Selfmonitoring in type 2 diabetes: A randomized trial of reimbursement policy. Diabet Med. 2006; $23: 1247-51$

6. Farmer A, Wade A, Goyder E, Yudkin P, French D, Craven A, et al. Impact of self monitoring of blood glucose in the management of patients with non-insulin treated diabetes: open parallel group randomised trial. BMJ. 2007;335:132.
7. Davis WA, Bruce DG, Davis TMD. Is Self-Monitoring of Blood Glucose Appropriate for All Type 2 Diabetic Patients? The Fremantle Diabetes Study. Diabetes Care. 2006;29:1764-70.

8. Tengblad A, Grodzinsky E, Lindstrom K, Molstad S, Borgquist L, Ostgren CJ. Self-monitoring of blood glucose and glycaemic control in type 2 diabetes. Scand J Prim Health Care. 2007; 25:140-6.

9. O'Kane MJ, Bunting B, Copeland M, Coates VE. Efficacy of self monitoring of blood glucose in patients with newly diagnosed type 2 diabetes (ESMON study): randomised controlled trial. BMJ. 2008;336:1174-7.

10. Faas A, Schellevis FG, Van Eijk JT. The efficacy of self-monitoring of blood glucose in NIDDM subjects. A criteria-based literature review. Diabetes Care. 1997;20:1482-6.

11. Coster S, Gulliford MC, Seed PT, Powrie JK, Swaminathan R. Selfmonitoring in type 2 diabetes mellitus: A meta-analysis. Diabet Med. 2000; 17:755-761.

12. Towfigh A, Romanova M, Weinreb JE, Munjas B, Suttorp MJ, Zhou A, et al. Self-monitoring of blood glucose levels in patients with type 2 diabetes mellitus not taking insulin: a meta-analysis. Am J Manag Care. 2008;14:468-75.

13. Davidson MB. Evaluation of self monitoring of blood glucose in non-insulin-treated diabetic patients by randomized controlled trials: little bang for the buck. Rev Recent Clin Trials. 2010;5:138-42.

14. Clar C, Barnard K, Cummins E, Royle P, Waugh N; Aberdeen Health Technology Assessment Group. Selfmonitoring of blood glucose in type 2 diabetes: systematic review. Health Technol Assess. 2010;14:1-140.

15. Welschen LM, Bloemendal E, Nijpels G, Dekker JM, Heine RJ, Stalman WA, et al. Self-monitoring of blood glucose in patients with type 2 diabetes who are not using insulin. Cochrane Database Syst Rev. 2005 Apr 18;(2):CD005060.

16. Clua Espuny JL, Puig Junoy J, Queralt Tomás ML, Palau Galindo A. Análisis coste-efectividad de la automonitorizacion de la glucosa sanguinea en diabeticos tipo 2. Gac Sanit. 2000;14:442-8.

17. Simon J, Gray A, Clarke P, Wade A, Neil A, Farmer A; Diabetes Glycaemic Education and Monitoring Trial Group. Cost effectiveness of self monitoring of blood glucose in patients with non-insulin treated type 2 diabetes: economic evaluation of data from the DiGEM trial. BMJ. 2008;336:1177-80. 
18. Cameron C, Coyle D, Ur E, Klarenbach S. Costeffectiveness of self-monitoring of blood glucose in patients with type 2 diabetes mellitus managed without insulin. CMAJ. 2010;182:28-34.

19. Sanyal C, Stephen D Graham SD, Cooke C. The relationship between type of drug therapy and blood glucose self-monitoring test strips claimed by beneficiaries of the Seniors' Pharmacare Program in Nova Scotia, Canada. BMC Health Serv Res. 2008, 8:111.

20. Clua Espuny JL, Puig Junoy J, Ciurana Roca E, García Bernal G, Monclus Benet JF, González Henares A, et al. Automonitorización de la glucosa sanguínea (MGS): evaluación de su prescripción y resultados en la diabetes tipo 2. Aten Primaria. 1999;24:316-25.

21. Lecomte P, Romonc I, Fosse S. Self-monitoring of blood glucose in people with type 1 and type 2 diabetes living in France: The Entred study 2001. Diabetes Metab. 2008;34:219-26.

22. Garzón G, Maganto A, Sacristán Rubio A, García P. La mitad de las tiras de glucemia capilar que utilizan nuestros pacientes tiene una dudosa indicación. Aten Primaria. 2005;36:405.

23. Gomes T, Juurlink DN, Shah BR, Paterson JM, Mamdani MM. Blood glucose test strips: options to reduce usage. CMAJ. 2010;182:35-8.

24. Belsey JD, Pittard JB, Rao S, Urdahl H, Jameson K, Dixon T. Self blood glucosemonitoring in type 2 diabetes. A financial impact analysis based on UK primary care. Int J Clin Pract. 2009;63:439-48.

25. Gulliford M, Latinovic R. Variations in glucose self-monitoring during oral hypoglycaemic therapy in primary care. Diabet Med. 2004;21:685-90.

26. Ricordeau P, Weill A, Vallier N, Bourrel R, Schwartz D, Guilhot J, et al. The prevalence and cost of diabetes in metropolitan France: what trends between 1998 and 2000?*. Diabetes Metab. 2003;29:497-504.

27. Centers for Disease Control and Prevention (CDC). Self-monitoring of blood glucose among adults with diabetes--United States, 1997-2006. MMWR Morb Mortal Wkly Rep. 2007;56:1133-7.

28. Patel H, Srishanmuganathan J, Car J, Majeed A. Trends in the prescription and cost of diabetic medications and monitoring equipment in England 19912004. J Public Health (Oxf). 2007;29:48-52.

29. Rathmann W, Haastert B, Icks A, Giani G. Trends in outpatient prescription drug costs in diabetic patients in Germany, 1994-2004. Diabetes Care. 2007;30:848-53.
30. Neutel CI, Campbell NR, Morrison HI. Trends in diabetes treatment in Canadians, 1994-2004. Chronic Dis Can. 2010;30:107-11.

31. Li R, Zhang P, Narayan KM. Self-monitoring of blood glucose before and after Medicare expansion among Medicare beneficiaries with diabetes who do not use insulin. Am J Public Health. 2008;98:358-64.

32. Dirección General de Farmacia y Productos Sanitarios. Uso adecuado de las tiras reactivas de glucosa en sangre en pacientes con diabetes mellitus. Valencia: Consellería de Sanitat; 2010.

33. Catalá Bauset M, Girbés Borrás J, coords Manual de educación diabetológica. Plan de Diabetes de la Comunidad Valenciana 2006-2010. Valencia: Conselleria de Sanitat de la Generalitat Valenciana; 2008

34. SMBG International Working Group. Self-monitoring of blood glucose in type 2 diabetes: an intercountry comparison. Diabetes Res Clin Pract. 2008;82:e15-8

35. Farmer A, Neil A. In response to 'variations in glucose self-monitoring during oral hypoglycaemic therapy in primary care'. Diabet Med. 2005;22:5112.

36. Burgers JS, Bailey JV, Klazinga NS, Van Der Bij AK, Grol R, Feder G; AGREE Collaboration. Inside guidelines: comparative analysis of recommendations and evidence in diabetes guidelines from 13 countries. Diabetes Care. 2002;25:1933-9.

37. García-Mayor R. Monitorización de la glucemia capilar en pacientes con diabetes mellitus de tipo2 no tratados con insulina. Med Clin (Barc). 2010;134:688-91.

38. Adams AS, Mah C, Soumerai SB, Zhang F, Barton MB, Ross-Degnan D. Barriers to self-monitoring of blood glucose among adults with diabetes in an HMO: a cross sectional study. BMC Health Serv Res. 2003;3(1):6.

39. Sanyal C, Graham SD, Cooke C, Sketris I, Frail DM, Flowerdew G. The relationship between type of drug therapy and blood glucose self-monitoring test strips claimed by beneficiaries of the Seniors' Pharmacare Program in Nova Scotia, Canada. BMC Health Serv Res. 2008;8:111.

40. Davis WA, Bruce DG, Davis TM. Is self-monitoring of blood glucose appropriate for all type 2 diabetic patients? The Fremantle Diabetes Study. Diabetes Care. 2006;29:1764-70. 
41. Kolb H, Martin S, Lodwig V, Heinemann L, Scherbaum WA, Schneider B. Are type 2 diabetes patients who self-monitor blood glucose special? The role of confounders in the observational ROSSO study. J Diabetes Sci Technol. 2009;3:1507-15.

42. Tuerk PW, Mueller M, Egede LE. Estimating physician effects on glycemic control in the treatment of diabetes: methods, effects sizes, and implications for treatment policy. Diabetes Care. 2008;31:869-73.

43. Kolb H, Martin S, Lodwig V, Heinemann L, Scherbaum WA, Schneider B. Are type 2 diabetes patients who self-monitor blood glucose special? The role of confounders in the observational ROSSO study. J Diabetes Sci Technol. 2009;3:1507-15.

44. Trinacty CM, Adams AS, Soumerai SB, Zhang F, Meigs JB, Piette JD, et al. Racial differences in longterm self-monitoring practice among newly drug-treated diabetes patients in an HMO. J Gen Intern Med. 2007;22:1506-13.

45. Tuerk PW, Mueller M, Egede LE. Estimating physician effects on glycemic control in the treatment of diabetes: methods, effects sizes, and implications for treatment policy. Diabetes Care. 2008;31(5):869-73.

46. Navarro Pérez J. Recomendaciones sobre el uso adecuado de las tiras reactivas de glucosa en sangre en pacientes con diabetes mellitus. FML. 2010; 14 (Supl1):3-5.

\section{Anexo 1}

\section{Grupo de Estudio de la Automonitoriza- ción Glucémica}

Diego Cano-Blanquer*, Jorge NavarroPérez (Departamento de Salud de ValènciaClínic-La Malva-rosa, Agencia Valenciana de Salud [AVS]), Pedro Cervera-Casino* (Departamento de Salud de Denia, AVS), Salvador Peiró-Moreno (Centro Superior de Investigación en Salud Pública), Mónica Mateu-García* (Departamento de Salud de Vinaròs, AVS), Amparo Barreda-Aznar*, $\mathrm{M}^{\mathrm{a}}$ Amparo Bonet-Dean* (Departamento de Salud de Castelló, AVS), Roberto Izquierdo-María*, Ma Magdalena Puig-Ferrer* (Departamento de Salud de La Plana, AVS), Jesús Larruga-Riera* (Departamento de Salud de Sagunt, AVS), Luis V García-Bataller (*) (Departamento de Salud de XàtivaOntinyent, AVS), Marisol Galeote-Mayor (*) (Departamento de Salud de Alcoi, AVS), María García-Gil (*) (Departamento de Salud de La Marina Baixa, AVS), Elías V. Salinas-Alemany (*) (Departamento de Salud de Alacant-Sant Joan d'Alacant, AVS).

(*) Sociedad Valenciana de Farmacéuticos de Atención Primaria. 